\title{
Identification and characterization of microRNAs involved in ascidian larval metamorphosis
}

\author{
Xiaoming Zhang ${ }^{1,2}$, Xiaozhuo Liu ${ }^{1}$, Chengzhang Liu' ${ }^{2,3}$, Jiankai Wei ${ }^{1,2}$, Haiyan Yu ${ }^{1}$ and Bo Dong ${ }^{1,2,4^{*}}$ (D)
}

\begin{abstract}
Background: Metamorphosis takes place within the life cycle of most marine invertebrates. The marine ascidian is a classical model to study complex cellular processes and underlying molecular mechanisms involved in its larval metamorphosis. The detailed molecular signaling pathways remain elusive, though extracellular signal-regulated kinases (ERKs) and c-Jun N-terminal kinase (JNK) have been revealed to regulate cell migration, differentiation, and apoptosis in ascidian larval organ regression and juvenile organ development. MicroRNAs (miRNAs) are small noncoding RNAs that modulate gene expression at the post-transcriptional level. Large numbers of miRNAs have been demonstrated to be involved in many developmental and metamorphic processes. However, the identification of miRNAs in ascidian larval metamorphosis has not yet been investigated.
\end{abstract}

Results: Totally, 106 known and 59 novel miRNAs were screened out through RNA-sequencing of three small RNA libraries from 18 to 21-h post-fertilization (hpf) tailbud embryos as well as from 42 hpf larvae (after tail regression) in Ciona savignyi. Expression profiling of miRNAs was confirmed by quantitative real-time PCR, showing that the expression levels of csa-miR-4040, csa-miR-4086, csa-miR-4055, csa-miR-4060, csa-miR-216a, csa-miR-216b, csa-miR-217, csa-miR-183, and csa-miR-92c were significantly higher in 42 hpf larvae, whereas those of csa-miR-4018a, csa-miR-4018b, and csa-miR-4000f were higher in 18 and 21 hpf embryos; then, their expression in 42 hpf larvae became significantly low. For these 12 miRNAs, whose expression levels significantly changed, we predicted their target genes through the combination of miRanda and TargetScan. This prediction analysis revealed 332 miRNA-target gene pairs that were associated with the ERK, JNK, and transforming growth factor beta signaling pathways, suggesting that the identified miRNAs are involved in the regulation of $C$. savignyi larval metamorphosis via controlling the expression of their target genes. Furthermore, we validated the expression of five selected miRNAs by northern blotting. Among the selected miRNAs, the expression patterns of csa-miR-4018a, csa-miR-4018b, and csa-miR-4000f were further examined by whole-mount in situ hybridization. The results showed that all three miRNAs were specifically expressed in a cell population resembling mesenchymal cells at the head and trunk part in swimming larvae but not in metamorphic larvae. Utilizing the luciferase assay, we also confirmed that miR-4000f targeted Mapk1, suggesting that the csa-miR-4018a/csa-miR-4018b/csa-miR-4000f cluster regulates larval metamorphosis through the Mapk1-mediated signaling pathway.

(Continued on next page)

\footnotetext{
* Correspondence: bodong@ouc.edu.cn

${ }^{1}$ Ministry of Education Key Laboratory of Marine Genetics and Breeding,

College of Marine Life Sciences, Ocean University of China, Qingdao 266003,

China

${ }^{2}$ Laboratory for Marine Biology and Biotechnology, Qingdao National

Laboratory for Marine Science and Technology, Qingdao 266237, China

Full list of author information is available at the end of the article
} 


\begin{abstract}
(Continued from previous page)
Conclusions: Totally, 165 miRNAs, including 59 novel ones, were identified from the embryos and larvae of C. savignyi. Twelve of them showed significant changes in expression before and during metamorphosis. In situ hybridization and northern blotting results revealed that three miRNAs are potentially involved in the signaling regulatory network for the migration and differentiation of mesenchymal cells in larval metamorphosis. Furthermore, the luciferase reporter assay revealed that Mapk 1 is a target of csa-miR-4000f. Our results not only present a list and profile of miRNAs involved in Ciona metamorphosis but also provide informative cues to further understand their function in ascidian larval metamorphosis.
\end{abstract}

Keywords: microRNAs, Ciona savignyi, Metamorphosis, Mesenchyme, Signaling pathways

\section{Background}

Larval metamorphosis is a key stage in ascidian development. Complex cellular processes and tissue morphogenesis are involved in larval tail regression and new organ formation in juveniles. The larval trunk mesenchyme derived from the A7.6 (trunk lateral cells, TLCs), B8.5 and B7.7, as well as B7.5 (trunk ventral cells, TVCs) blastomeres of the 110-cell embryo is essential for making diverse adult organs including tunic cells, blood cells, muscles, and the heart [1]. The differentiation and migration of mesenchymal cells are highly coordinated spatially and temporally by a complex combination of signaling pathways, yet the underlying mechanisms remain unclear. The signaling pathways involved in Ciona larval tail regression initiation and propagation have been revealed in the past years. The epithelial growth factor (EGF) pathway plays an important role in early larval metamorphosis [2-4]. The mitogen-activated protein (MAP) kinases ERK and c-Jun N-terminal kinase (JNK) activate and regulate their downstream gene expression in tail tissues to drive the initiation of tail regression $[5,6]$. Nitric oxide regulates tail regression through the JNK signaling pathway $[7,8]$. However, little is known regarding molecular regulatory mechanisms on the origin and formation of juvenile tissues during metamorphosis.

miRNAs are a class of endogenous small non-coding RNA molecules that regulate the post-transcriptional expression of target genes by translational repression or transcript cleavage. They are involved in numerous biological processes, such as cell proliferation, apoptosis, growth, and migration [9-11]. Previous studies have shown that miRNAs are necessary for regulating metamorphosis in insects [12, 13]. Hundreds of miRNAs have been identified or predicted in tunicates, including Oikopleura dioica [14], Ciona intestinalis [15, 16], Didemnum vexillum [17], and Halocynthia roretzi [18]. Comparing miRNAs within most phyla, tunicates gained many novel non-conserved miRNA families [19]. Within the tunicate lineage, Ciona retained the major chordate miRNA families and most $C$. intestinalis miRNA families are conserved in C. savignyi [16]. Although numerous ascidian miRNAs have been identified, few miRNAs have been experimentally validated, and the biological functions of most miRNAs remain poorly understood. Thus far, miR-124 has been demonstrated to promote nervous system development by interacting with the Notch pathway in C. intestinalis embryogenesis [20]. However, little is known about the role of miRNAs in ascidian larval metamorphosis.

In the present study, three miRNA libraries from different stages of C. savignyi were constructed, and 106 known and 59 novel miRNAs were identified. The expression profiles of 78 miRNAs were validated, revealing that the expressional levels of 12 miRNAs showed significant differences before and during metamorphosis. In addition, the target genes of these 12 miRNAs were predicted. Furthermore, the expression patterns of three miRNAs were examined by in situ hybridization and, it was found that they were specifically expressed in trunk mesenchymal cells, indicating that they participated in new tissue formation in Ciona larval metamorphosis.

\section{Results}

\section{Identification of miRNAs from three miRNA libraries of C. savignyi larvae}

To identify miRNAs that participated in C. savignyi metamorphosis, three miRNA libraries from larvae before (18 and $21 \mathrm{~h}$ post fertilization, hpf) and during (42 hpf) metamorphosis were constructed and sequenced. The total reads were $34,683,647$. Nucleotide length distributions of sequenced small RNAs were analyzed by Solexa sequencing. Most sequenced small RNAs were 21-30 nucleotides in length (Fig. 1a). After comparing sequenced small RNA sequences with the existing $C$. savignyi mRNA database, 11,283,372 reads were mapped on the reference sequence. Then, 2,544,546 reads, which encode mRNA, rRNA, tRNA, snoRNA, repeats, introns, and exons, were removed (Fig. 1b). The remaining $8,738,826$ reads were further analyzed through comparison with known miRNAs (miRNA precursors and mature miRNAs) in miRBase 13.0. A total of 106 known miRNAs (Additional file 1: Table S1), which were homologous to identified miRNAs from other species, and 59 novel ones (Additional file 2: Table S2) that did not 

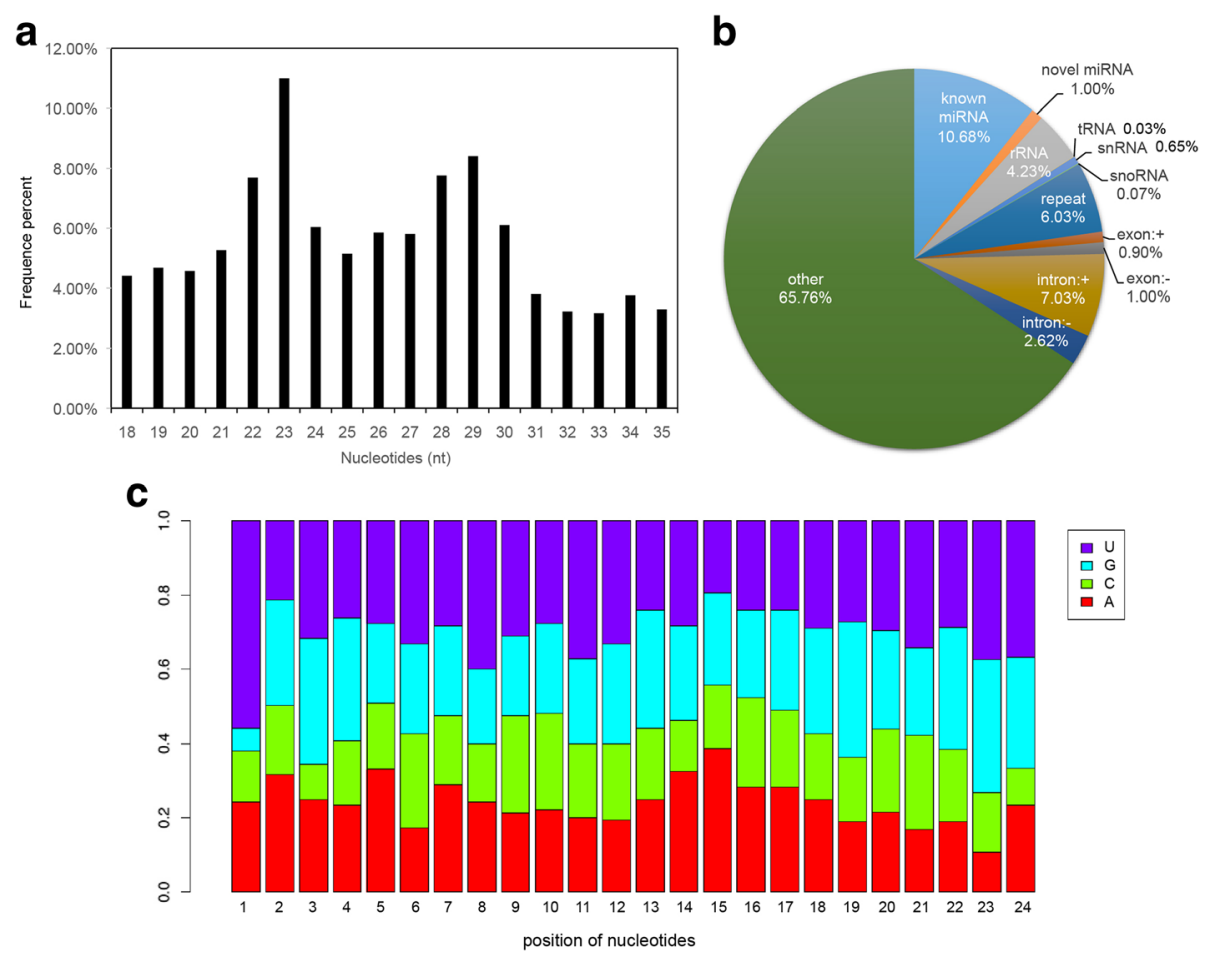

Fig. 1 Characterization of sequenced data from three miRNA libraries of Ciona savignyi larvae. a Size distribution of sequenced small RNAs. $\mathbf{b}$ The classification and abundance of small RNA sequencing reads. Known miRNA, miRNAs that align to miRNAs in miRBase; novel miRNA, miRNAs that do not match any known miRNA/RNA sequence; rRNA, ribosomal RNA; tRNA, transfer RNA; snRNA, small nuclear RNA; snoRNA, small nucleolar RNA; repeat, repeat associate RNA; exon:+, exon in the DNA sense strand; exon:-, exon in the DNA antisense strand; intron:+, intron in the DNA sense strand; intron:-, intron in the DNA antisense strand; other, sequences do not match above classifications. c The frequency of miRNA nucleotide bases $(A / U / C / G)$ at each position. Purple represents $U$, blue represents $G$, green represents $C$, and red represents $A$

match any known miRNA in miRBase were identified. Nucleotide base $(\mathrm{A} / \mathrm{U} / \mathrm{C} / \mathrm{G})$ analysis showed that the dominant first preferred nucleotide base at the first position was U (Fig. 1c).

\section{Origin and evolution of identified miRNAs}

A total of 150 miRNA families were identified for $C$. savignyi, including 93 conserved and 57 novel families.
To trace the evolutionary origin of these miRNAs, miRNA conservation across the phylogeny of metazoans was explored (Fig. 2). miR-10 was acquired at the base of Eumetazoa. Eighteen families evolved during the origin of Nephrozoa (miR-1, 7, 8/200, 25/92, 31, 34, 46/ $281,96,124,125,133,153,183,184,219,281,375$, and let-7). One family was acquired at the base of Deuterostomia (miR-367). Four families evolved at the origin of

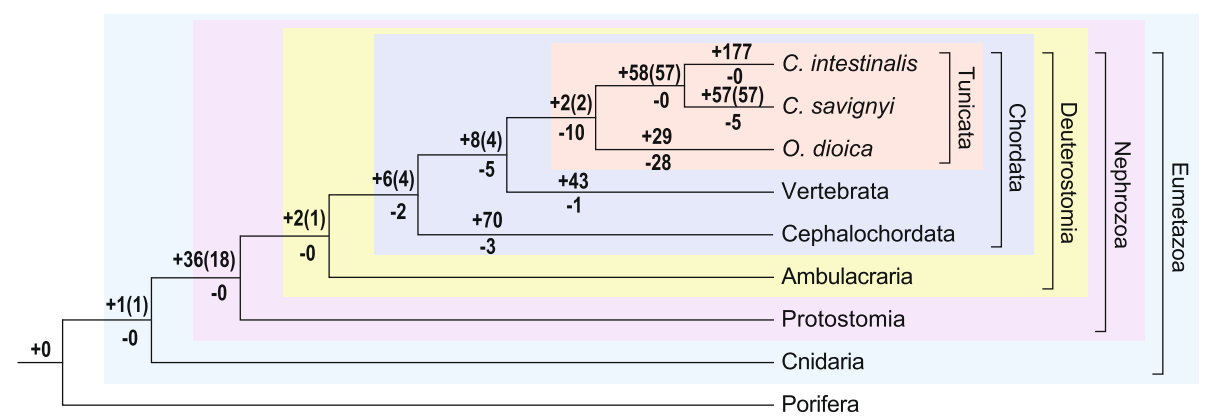

Fig. 2 Evolutionary history of tunicate miRNAs. C. savignyi miRNAs were compared with miRNAs of 16 other animal species from miRBase. Only miRNAs supported by experimental evidence were used. An miRNA family was defined as acquired at the origin of a taxon if it is shared by at least two daughter lineages of the taxon but if is absent in all ancestor and sister lineages. At the base of each taxon, numbers are shown for the miRNA families acquired ( + ) and lost (-) during the origin of this taxon. A number is also given in parentheses to indicate the acquired families that are present in C. savignyi (not lost in evolution) 
Chordata (miR-135, 141, 217, 4057). The miR-1497 and 1502 families evolved at the origin of Tunicata, and 58 miRNA families were added at the base of Ciona.

\section{Expression profiles of the identified miRNAs}

To screen those miRNAs that potentially participated in C. savignyi larval metamorphosis, the expression profiles of the identified miRNAs at different stages were validated using quantitative real-time PCR (qPCR). The heat map showed the expressional level of the identified miRNAs (Fig. 3a). The expression of miRNAs from 18 and 21 hpf embryos did not generally show any significant difference. However, for some miRNAs from $42 \mathrm{hpf}$ larvae, their expression was significantly different compared with that at the earlier stages. Further characterized results showed that the expression levels of nine miRNAs (csa-miR-4040, csa-miR-4086, csa-miR-4055, csa-miR4060, csa-miR-216a, csa-miR-216b, csa-miR-217, csa-miR183 , and csa-miR-92c) significantly increased at $42 \mathrm{hpf}$ compared with those at 18 and $21 \mathrm{hpf}(P<0.01)$ (Fig. 3b). Conversely, the expression levels of three miRNAs (csa-miR-4018a, csa-miR-4018b, and csa-miR-4000f) significantly decreased in metamorphic larvae $(P<0.01)$ (Fig. 3c), suggesting that they play vital roles in C. savignyi larval metamorphosis.

\section{Interaction between the identified miRNAs and their target gene predictions}

For 12 miRNAs with either significantly higher or lower expression during larval metamorphosis, their target genes were predicted utilizing miRanda and TargetScan. Candidate target genes were from RNA-Seq data obtained from 18, 21, and $42 \mathrm{hpf}$ embryos/larvae and the ENSEMBL database. Those genes overlapping in the prediction by the two softwares or their expressions in RNA-Seq negatively correlated with the 1 of the $12 \mathrm{miR}$ NAs were picked as target genes. A total of 332 miRNAtarget gene pairs were found and displayed by Cytoscape 3 in Fig. 4a. The results showed a complex network consisting of differentially expressed miRNAs and their target genes. Among them, csa-miR-92c targeted 114 genes including mitogen-activated protein kinase kinase (MKK) and mitogen-activated protein kinase phosphatase (MKP), which belong to the MAPK signaling pathway.
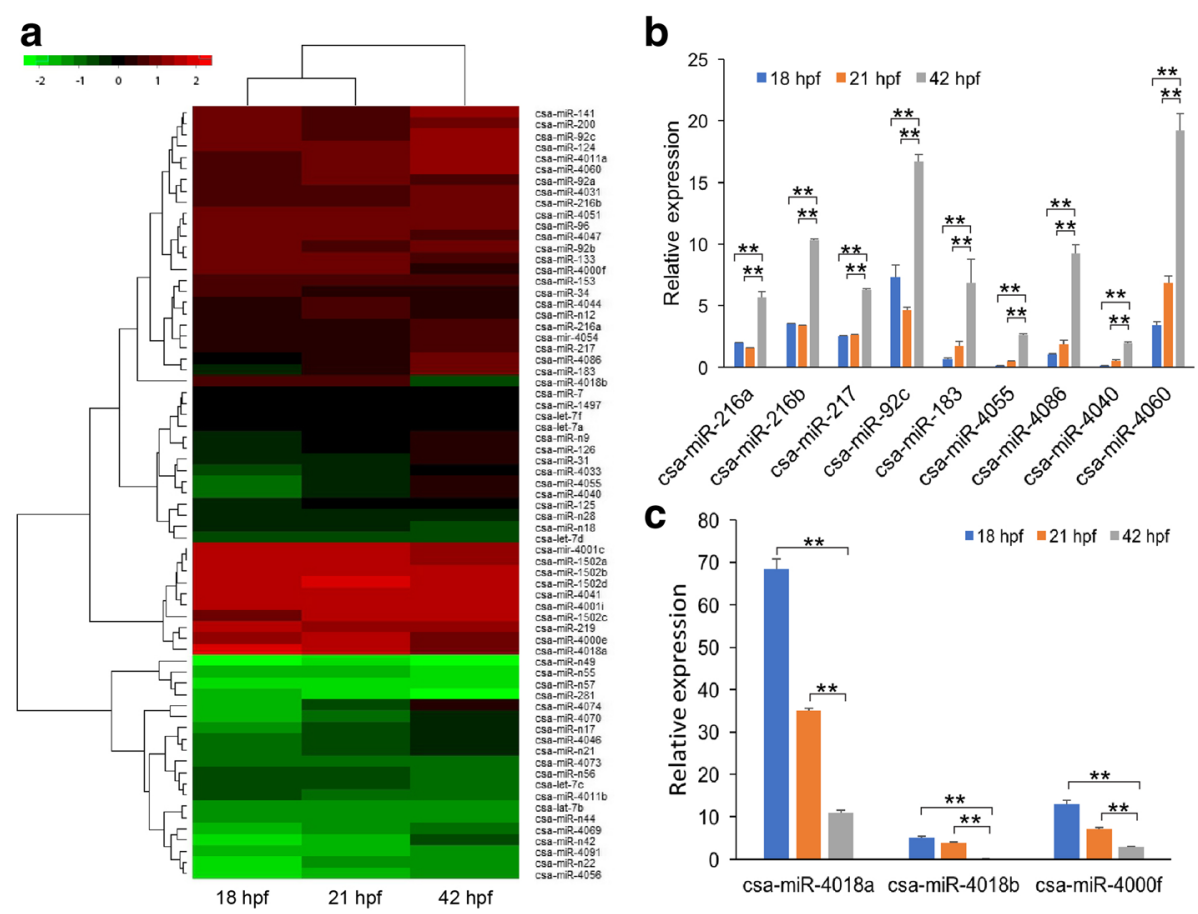

Fig. 3 miRNA expression profiles before and after C. savignyi larval metamorphosis. a Heat map of relative expression levels of miRNA of C. savignyi larvae at 18, 21, and $42 \mathrm{hpf}$ stages detected by qPCR. Each row represents an miRNA, and each column represents a specific stage indicated at the bottom. The expression of miRNA was hierarchically clustered on the left, and the sample clustering was presented at the top. The values shown are averages of independent experiments performed in triplicate. The color bar depicts the color contrast level of the heat map. Green or red on the heat map indicates a decrease or increase in miRNA levels, respectively. $\mathbf{b}-\mathbf{c}$ The expression levels of upregulated miRNAs (b) and downregulated miRNAs (c) at $42 \mathrm{hpf}$ were identified by qPCR. The relative expression of each miRNA was calculated using the delta-delta Ct method (2 $\left.2^{-\Delta \Delta C t}\right)$ and normalized to U6 snRNA. Results represented the mean of triplicate assays with standard error (mean \pm SD). Significance of relative expression at different stages was determined using Student's $t$-test. Asterisks $\left(^{* *}\right)$ represented statistical significance at $P<0.01$. Blue, orange and gray bars represent expression levels at 18,21, and $42 \mathrm{hpf}$ of each miRNA, respectively 

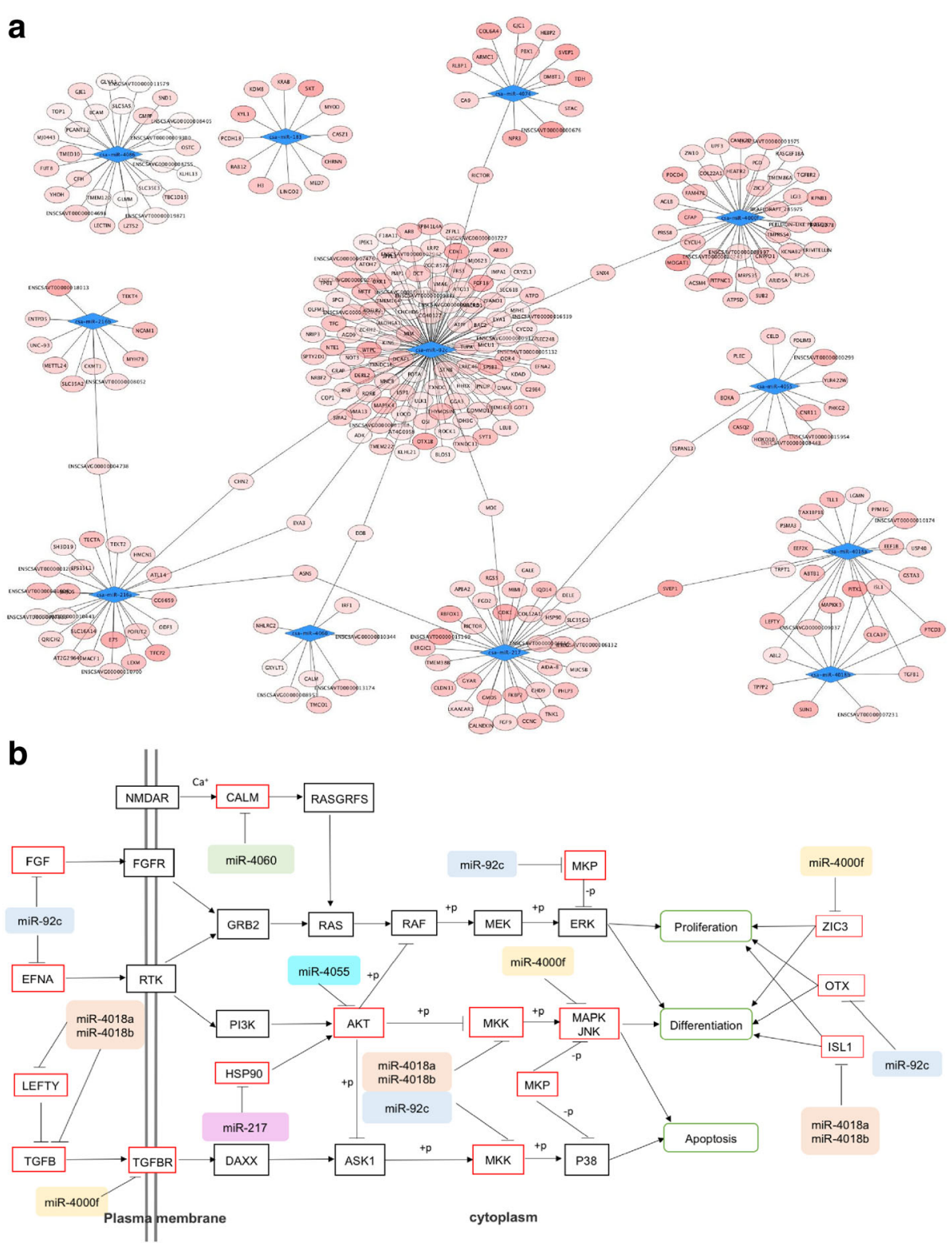

Fig. 4 The interactions of identified miRNAs and their target gene prediction. a The interactions of 12 miRNAs and their target genes were visualized as a network using Cytoscape3.4. The target genes displayed in the network included the overlapping ones predicted by miRanda and TargetScan and those genes whose expression levels on performing RNA-Seq were negatively correlated with the expression of miRNAs. Nodes of blue diamonds and pink ovals represented miRNAs and their target genes, respectively. Gradient pink color in the nodes of the target reflects different free energy levels of the miRNA/target duplex (from dark pink to light pink for lower energy to higher energy). Black solid lines between nodes indicated the interrelationships of miRNAs. b Signal pathways related to C. savignyi metamorphosis targeted by miRNAs. Red line rectangles represent target genes; black line rectangles represent genes in a pathway; colored filled rectangles represent different miRNA; green line rounded rectangles represent biological processes; black arrows represent positive regulation; black line-bar represents negative regulation

Transforming growth factor (TGF) beta 1 (TGF- $\beta 1)$, sorting nexin 4 , pituitary homeobox, and others were targeted by two miRNAs, and the target genes of miR-4018a and miR-4018b significantly overlapped.

Furthermore, KEGG pathway enrichment analysis was performed. The results showed that the MAPK, Rasrelated protein 1 (Rap1), Ras, and TGF- $\beta$ signaling pathways were involved in the target gene database (Fig. 4b).
In the MAPK signaling pathway, csa-miR-4018a and csa-miR-4018b targeted MKK and csa-miR-92c targeted MKK and MKP. In the TGF- $\beta$ signaling pathway, csamiR-4018a and csa-miR-4018b commonly targeted the left-right determination factor and miR-4000f targeted the TGF- $\beta$ receptor. In addition, miR-92c targeted FGF and Ephrin-A, which are upstream of the Rap1 and Ras signaling pathway. 
It is worth noting that zinc finger protein of the cerebellum 3, orthodenticle homeobox, and insulin gene enhancer protein, which regulate pluripotency in stem cells [21-23], were targeted by csa-miR-4000f, csa-miR-92c, and csa-miR-4108a/csa-miR-4108b, respectively, revealing that they might control larval stem cell differentiation during metamorphosis. In addition, genes related to lysosome, endoplasmic reticulum, phagosome, endocytosis, and autophagy regulation were significantly enriched in KEGG results (Table 1), indicating that vesicle trafficking processes are involved in larval metamorphosis.

\section{Validation of the identified miRNA expression levels}

The above screened 12 miRNAs included seven miRNAs which were annotated from bioinformatics analysis [16], but were not previously verified by experiments. To further validate the existence and expression of those identified miRNAs, northern blotting of seven miRNAs was conducted using RNA extracted from C. savignyi. Locked nucleic acid (LNA) probes were designed to detect miRNAs.
U6 snoRNA was used as the loading control (Fig. 5a). Five probes from csa-miR-4040, csa-miR-4086, csa-miR-4000f, csa-miR-4018a, and csa-miR-4018b showed bands. The size of each band was consistent with that of mature miRNAs (21-24 nucleotides in length) (Fig. 5b and Additional file 3: Figure S1), indicating that these five miRNAs exist in the $C$. savignyi genome and that the mature ones are expressed during larval metamorphosis.

\section{Expression patterns of miRNAs downregulated during metamorphosis}

For miRNAs confirmed by northern blotting, their expression pattern was further examined in embryos and larvae utilizing whole-mount in situ hybridization. At 21 hpf, the expression of csa-miR-4018a, csa-miR-4018b, and csa-miR-4000f was detected. The results showed that they were all distributed in a scattered cell population at the head and trunk part in tailbud embryos (Fig. 6). At a later stage (31 hpf), stronger expression signals of all three miRNAs moved to the trunk part.

Table 1 KEGG pathway analysis of transport and catabolism

\begin{tabular}{|c|c|c|c|c|}
\hline Pathway & Target Gene & $\mathrm{KO} I \mathrm{D}$ & CDNA ID & miRNA \\
\hline Endoplasmic & STT3 & K07151 & ENSCSAVG00000004164 & csa-miR-4000f \\
\hline \multirow[t]{7}{*}{ Reticulum } & CALR & K08057 & ENSCSAVG00000000885 & csa-miR-217 \\
\hline & MBTPS1 & K08653 & ENSCSAVG00000005178 & csa-miR-4000f \\
\hline & HYOU1 & K09486 & ENSCSAVG00000003312 & csa-miR-92c \\
\hline & HSP90B & K09487 & ENSCSAVG00000010760 & csa-miR-217 \\
\hline & PDIA6 & K09584 & ENSCSAVG00000010980 & csa-miR-92c \\
\hline & EDEM1 & K10084 & ENSCSAVG00000000652 & csa-miR-92c \\
\hline & SEC23 & K14006 & ENSCSAVG00000011363 & csa-miR-92c \\
\hline \multirow[t]{6}{*}{ Phagosome } & ATPeV1H & K02144 & ENSCSAVG00000000896 & csa-miR-92c \\
\hline & ATPeV1A & K02145 & ENSCSAVG00000005752 & csa-miR-92c \\
\hline & ATPeVOD & K02146 & ENSCSAVG00000001895 & csa-miR-92c \\
\hline & ATPeV1F & K02151 & ENSCSAVG00000011191 & csa-miR-92c \\
\hline & CALR & K08057 & ENSCSAVG00000000885 & csa-miR-217 \\
\hline & SEC61B & K09481 & ENSCSAVG00000002561 & csa-miR-92c \\
\hline \multirow[t]{4}{*}{ Endocytosis } & $\mathrm{SH} 3 \mathrm{G}$ & K11247 & ENSCSAVG00000002074 & csa-miR-216a \\
\hline & ARFGAP2/3 & K12493 & ENSCSAVG00000001512 & csa-miR-92c \\
\hline & CCDC53 & K18463 & ENSCSAVG00000005922 & csa-miR-92c \\
\hline & VPS29 & K18467 & ENSCSAVG00000005941 & csa-miR-92c \\
\hline \multirow[t]{6}{*}{ Lysosome } & GALC & K01202 & ENSCSAVG00000002286 & csa-miR-92c \\
\hline & ATPeV1H & K02144 & ENSCSAVG00000000896 & csa-miR-92c \\
\hline & ATPeVOD & K02146 & ENSCSAVG00000001895 & csa-miR-92c \\
\hline & AP1M & K12393 & ENSCSAVG00000004175 & csa-miR-92c \\
\hline & LGMN & K01369 & ENSCSAVG00000006624 & csa-miR-4018a \\
\hline & GGA & K12404 & ENSCSAVG00000004489 & csa-miR-92c \\
\hline \multirow[t]{2}{*}{ Autophagy } & ULK1/2/3 & K08269 & ENSCSAVG00000003676 & csa-miR-92c \\
\hline & ATG13 & K08331 & ENSCSAVG00000003971 & csa-miR-92c \\
\hline
\end{tabular}




\section{b}

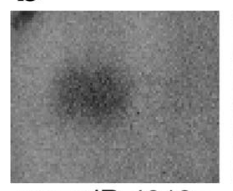

csa-miR-4018a a

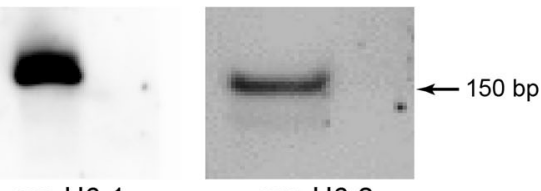

csa-U6-1
csa-U6-2
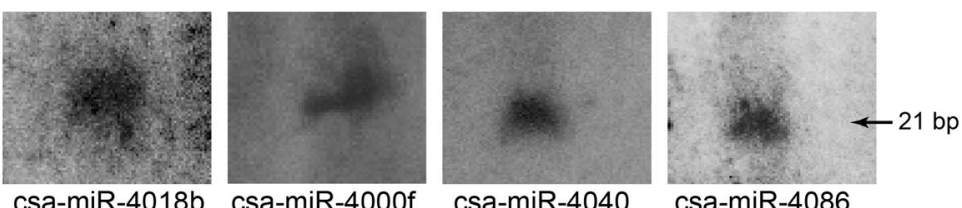

csa-miR-4040

Fig. 5 Validation of the identified miRNA existence and their expression by northern blotting. a U6 snRNA served as the loading control. Csa-U6-1 and csa-U6-2 using total RNA extracted from embryos and adult total RNA, respectively. b Northern blot analysis of csa-miR-4018a, csa-miR-4018b, csa-miR-4040, csa-miR-4086, and csa-miR-400of was performed in denaturing gels using LNA probes. The size of mature miRNAs was approximately 21 nucleotides

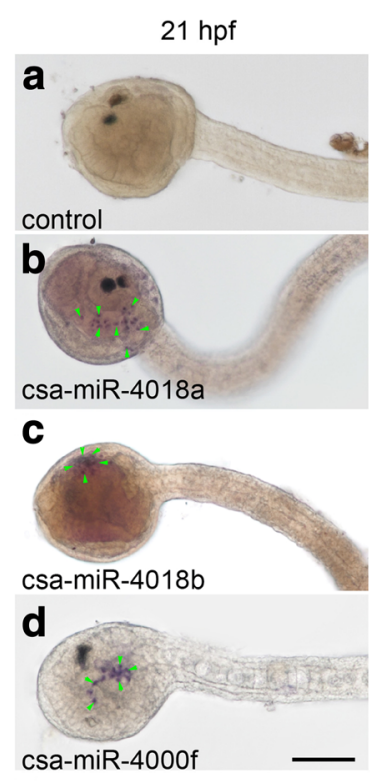

e

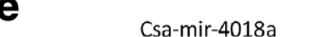

$31 \mathrm{hpf}$
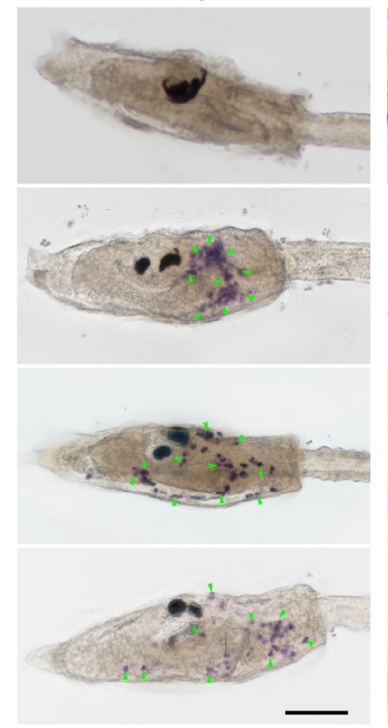

Csa-mir-4000f

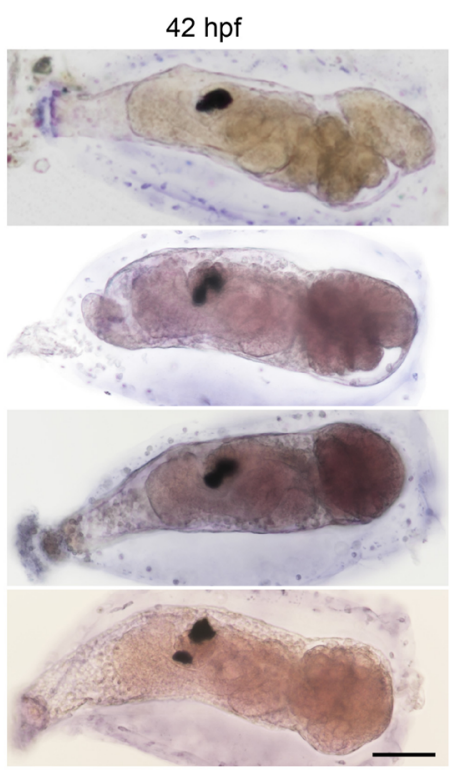

Csa-mir-4018b

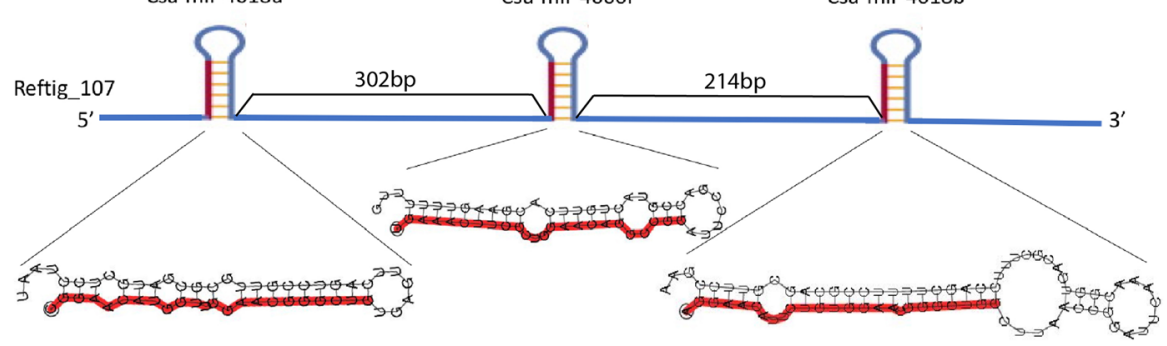

Fig. 6 Expression patterns of three miRNAs detected by whole-mount in situ hybridization. Embryos and larvae at 21, 31, and 42 hpf were hybridized with LNA probes of scrambled control (a), csa-miR-4018a (b), csa-miR-4018b (c), and csa-miR-4000f (d). The developmental stages are indicated in column headings. Hybridized signals are indicated by green arrowheads. The expression signals of csa-miR-4018a, csa-miR-4018b, and csa-miR-4000f were specifically detected in a scattered cell population at 21 and 31 hpf, and their expression signals were stronger at 31 hpf but weaker at $42 \mathrm{hpf}$. (e). Schematic representation of the genomic localization of the precursors of csa-miR-4018a, csa-miR-4018b, and csa-miR-4000f. The numbers on the blue line represent the distance between two pre-miRNAs. Scale bars represent $100 \mu \mathrm{m}$ 
During larval metamorphosis (42 hpf), a weak signal could be examined for the csa-miR-4018a probe (Fig. 6b). There were no detectable signals for the csa-miR-4018b and csa-miR-4000f probes (Fig. 6c-d). The expression patterns of the three miRNAs were similar and resembled that of mesenchymal cells [1]. Through a search of the genome scaffold, csa-miR-4018a, csa-miR-4018b, and csa-miR-4000f formed an miRNA cluster, consecutively located in scaffold reftig_107 (Fig. 6e), indicating that csa-miR-4018a, csa-miR-4018b, and csa-miR-4000f share similar regulatory elements. Significant expression changes of the three miRNAs in mesenchymal cells suggest that they play important roles in larval metamorphosis.

\section{Identification of Mapk1 as the target gene of miR-4000f} miRNA target prediction suggested that both csa-miR4018a and csa-miR-4018b targeted Mapkk3 (Additional file 4: Figure S2A) and that csa-miR-4000f targeted Mapk1 (Fig. 7a) through seed sequences. To investigate how the csa-miR4018a/csa-miR-4018b/csa-miR-4000f cluster functions in larval metamorphosis, above prediction result was further experimentally confirmed through the luciferase assay. miRNA mimics or the miR-control was co-transfected into HEK293T cells with either its target gene 3'-UTR or 3'UTR mutants, respectively. Luciferase activities were then measured at $48 \mathrm{~h}$ after each transfection. The results showed that normalized luciferase activities in cells co-transfected with csa-miR-4000f and pmirGLO-Mapk1-3'-UTR decreased $42 \%$ compared with that in miR-control and pmirGLO-Mapk1-3' - UTR co-transfected cells, whereas the relative luciferase activities in csa-miR-4000f and pmirGLOMapk1-3'-UTR mutant co-transfected cells did not significantly decrease (Fig. 7b), indicating that Mapk1 is the target gene of csa-miR-4000f.

We also examined binding activities between csa-miR4018a or csa-miR-4018b and Mapkk3 utilizing the same approach. No significant reduction in luciferase activity appeared in csa-miR-4018a or csa-miR-4018b and pmirGLO-Mapkk3-3'-UTR co-transfected cells (Additional file 4: Figure S2B). These results suggest both csa-miR4018a and csa-miR-4018b do not target Mapkk3.

\section{Discussion}

In this study, 106 conserved miRNAs and 59 novel miRNAs were identified through the screening of three small RNA libraries from C. savignyi larvae before and during metamorphosis. The number of identified miRNAs was less than the number found in a previous study [16]. The reason was partly due to the utilization of embryos/ larvae at three stages $(18,21$, and $42 \mathrm{hpf})$ to construct libraries in our experiments. It has been found that lower levels of miRNA are expressed in late embryos and adults than in unfertilized eggs and early embryos [16].

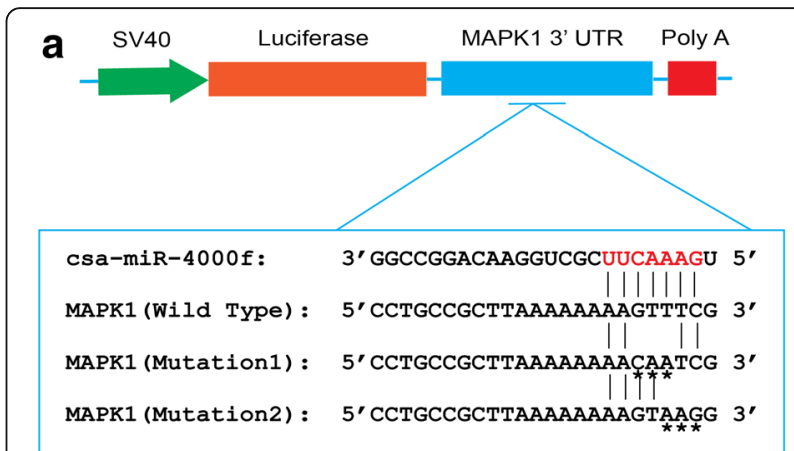

b

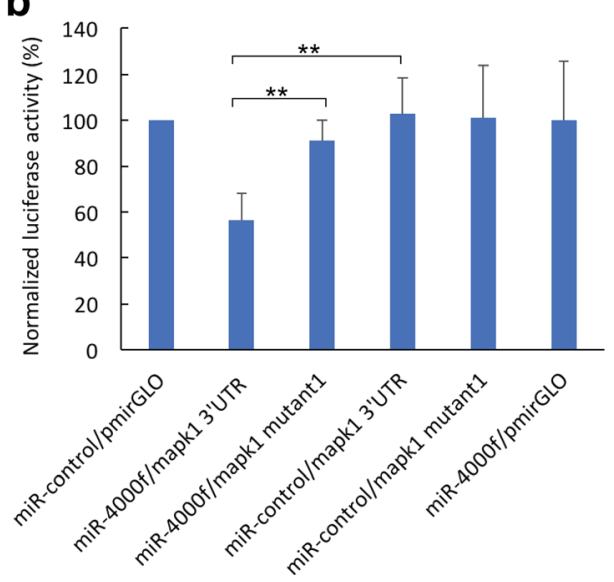

Fig. 7 Csa-miR-4000f targets the Mapk1 by interacting with its 3'UTR. a Schema of luciferase reporter constructs carrying the $3^{\prime}-$ UTR regions of target genes used in luciferase assays. Mapk1-3'-UTR or Mapk1-3'-UTR mutants were cloned into the downstream luciferase open reading frame of the pmirGLO dual luciferase vector. Sequence alignments of csa-miR-4000f and its predicted target gene Mapk13'-UTR or mutants of the Mapk1-3'-UTR are displayed in the blue line box. Asterisks $\left(^{*}\right)$ under letters indicate the mutant nucleotide in the Mapk1-3'-UTR mutant constructs. b Relative luciferase activity in HEK-293T cells co-transfected with pmirGLO-Mapk1-3'-UTR or pmirGLO-Mapk1-3'-UTR mutants or the empty vector pmirGLO and either csa-miR-4000f mimics or miR-control. Firefly luciferase values were normalized for transfection with Renilla luciferase activity. Student's $t$-test was used to evaluate the significance of luciferase data. Asterisks $\left.{ }^{(*}\right)$ represented statistical significance at $P<0.01$

Although species belonging to Tunicata have retained most deuterostome miRNAs, there were a significant number of miRNA families (miR-4, 19, 22, 50/190, 129, $137,193,210,315,365)$ that were lost at the base of the tunicate lineage compared with the only two families (miR-1497 and 1502) acquired during evolution. This is a rare phenomenon considering that miRNAs are seldom lost during evolution [24]. Moreover, another 28 conserved miRNAs seemed to be lost in the freeswimming tunicate $O$. dioica. The miRNA families of Ciona and $O$. dioica were surprisingly different as they shared only two tunicate-acquired miRNA families, while 61 and 31 new miRNAs originated in each branch, respectively, during the diversification of the two groups. 
As the evolution of miRNA is believed to correlate with morphological complexity [25], the dynamic miRNA repertoire in the tunicate lineage might be involved in the degeneration and rearrangement of body plans during metamorphosis. Expression profiling of miRNAs using qPCR showed that the expression of the three miRNAs acquired at the origin of Chordata (miR-141, 217) and the vertebrata plus tunicata (miR-126) lineages were all significantly upregulated at $42 \mathrm{hpf}$ larvae, whereas the two Tunicata-acquired miRNAs (miR-1497, 1502) did not show such a pattern.

Among 165 identified miRNAs, 78 were further investigated by qPCR to confirm their temporal expression profile before and during C. savignyi larval metamorphosis. Twelve miRNAs were validated to be significantly either up- or downregulated at $42 \mathrm{hpf}$, indicating that they were involved in the regulation of larval metamorphosis. Upregulated miRNAs might function to inhibit their targets, such as MKP, which could hinder the progression of developmental events such as cell apoptosis. Metamorphosis involves numerous rapid morphogenetic movements and physiological changes and is regulated by several complicated signal pathways and gene networks. Thus, the lower expression of some miRNAs during metamorphosis could trigger their target genes, such as MKK, and result in obvious alterations in subsequent larval survival and adult tissue development.

Previously identified Ciona miRNAs were involved in unfertilized egg, embryo, and adult development [16]. In the present study, to the best of our knowledge, we have, for the first time, identified miRNAs that have potentially regulated larval metamorphosis. To uncover the biological roles of miRNAs in metamorphosis, we predicted their target genes and performed KEGG pathway enrichment analysis. A total of 332 miRNA-target gene pairs were predicted by miRanda and TargetScan. Some target genes, such as EGF and Sushi, have been reported to be important molecules during larval metamorphosis $[4,5]$. In the present study, the MAPK, Rap1, Ras, EGF, and TGF- $\beta$ signaling pathways were identified to be involved in the target gene database. Some elements of these pathways have been found during Ciona larval metamorphosis $[6,26]$, suggesting that miRNAs interact with their target genes to regulate tissue rearrangement during larval metamorphosis. In addition, several pathways relevant to vesicle formation, transportation, and secretion were identified. Recently, exosomes have been widely reported to mediate cell-to-cell communication. miRNAs are one of the important cargos of exosomes that play crucial roles in diverse developmental events [27, 28]. Thus, our data revealed that miRNAs might function in larval metamorphosis via extracellular exosome trafficking.

The cell population in which csa-miR-4018a, csa-miR4018b, and csa-miR-4000f were expressed resembled mesenchymal cells in the Ciona larval trunk [1], which is essential for the formation of the heart, muscles, blood, and juvenile organs in metamorphic larvae. Ascidian larvae contain three lines of mesenchymal cells TLCs, TVCs, and mesenchyme [1]. At 31 hpf, csa-miR-4018a, csa-miR4018b, and csa-miR-4000f showed different expression patterns. This could be caused either by the migration of mesenchymal cells in the trunk during larval development or by the expression of these three miRNAs in different subpopulations of the mesenchyme in ascidian larvae. In mammals, mesenchymal stem cells (MSCs) are multipotent stromal cells that differentiate into various cell types [29]. Ciona miRNAs expressed in mesenchymal cells might regulate new tissue development by interacting with their target genes. KEGG pathway enrichment analyses showed that their target genes were included in the genes of the MAPK, Rap1, Ras, and TGF- $\beta$ signal pathways. We further confirmed that Mapk1 is the target of csa-miR-4000f. During early embryogenesis in ascidians, MAPK-mediated FGF signaling is required to induce mesenchyme cell fate through the activation of different genes [30-32]. In Ciona larval metamorphosis, the MAPK pathway has been demonstrated to be required for tail regression $[5,6]$. Our current results suggested that the csa-miR-4018a/csa-miR4018b/csa-miR-4000f cluster regulates larval metamorphosis through the MAPK1-mediated signaling pathway.

Recent studies have implicated that exosomes are the key effectors of MSC function; exosomes are able to carry miRNAs and are released by MSCs to facilitate diverse functions [29, 33, 34]. Thus, we hypothesized that Ciona mesenchymal cells regulate adult tissue proliferation and differentiation by transferring miRNAs to recipient cells through the release of exosomes. Csa-miR-4018a, csamiR-4018b, and csa-miR-4000f were located adjacent to each other on the same chromosome and formed a miRNA cluster. Generally, high sequence homology between the miRNAs in a cluster permit both common and unique mRNA targets. These miRNA targets lie within the same pathway, thereby allowing these miRNAs to regulate several components of a cellular process [35]. The similar expression patterns of these three miRNAs (Fig. 6) indicated that the members of this miRNA cluster were transcribed as a single precursor. It is interesting to further investigate how these three miRNAs contribute to larval metamorphosis by regulating cell differentiation and migration in the near future.

\section{Methods}

\section{Animals, embryos, and larvae}

C. savignyi adults were collected from the coast of Qingdao, China. The animals were kept and acclimated in aerated seawater for three days under constant light to accumulate gametes. Eggs were then dissected and mixed with seawater with sperm from other individuals. Five minutes after 
fertilization, eggs were washed with seawater through a nylon filter to remove sperm and debris. Embryos were cultured at $16{ }^{\circ} \mathrm{C}$.

\section{Small-RNA library construction and sequencing}

Ascidian embryos and larvae were collected at 18, 21, and $42 \mathrm{hpf}$ and were then immediately frozen in liquid nitrogen. Total RNA was extracted with RNAiso Reagent (Takara). Totally, $3 \mu \mathrm{g}$ of total RNA per sample was used as input material for the construction of small RNA libraries. Libraries were generated using NEBNext ${ }^{\oplus}$ Multiplex Small RNA Library Prep Set for Illumina ${ }^{\circledast}$ (NEB) following the manufacturer's instructions and were sequenced on an Illumina Hiseq 2500/ 2000 platform at Novogene, China.

\section{qPCR}

Reverse transcription was carried out using the Mir- $\mathrm{X}^{\text {тм }}$ miRNA First-Strand Synthesis Kit (Clontech). QPCR amplification was performed using the Mir-X miRNA qPCR SYBR Kit (Clontech) on LightCycler 480 (Roche). The reaction conditions were as follows: $95{ }^{\circ} \mathrm{C}$ for $10 \mathrm{~min}, 40$ cycles of $95^{\circ} \mathrm{C}$ for $15 \mathrm{~s}$, and $60{ }^{\circ} \mathrm{C}$ for $1 \mathrm{~min}$. U6 snRNA served as an internal normalization control. The entire sequence of mature miRNA was used as a miRNA-specific 5' primer. Data were analyzed using the comparative $\mathrm{Ct}$ method, and statistical analyses were performed using paired Student's $t$-tests.

\section{Northern blot}

Total RNA $(8-20 \mu \mathrm{g})$ from $21 \mathrm{hpf}$ embryos and adults were isolated using RNAiso Reagent (Takara) and electrophoresed in a denaturing $12 \%$ polyacrylamide gel and transferred onto a Hybond-N+ membrane (GE Healthcare). miRNA probes (Additional file 5: Table S3) were DIGlabeled LNA-modified probes synthesized by Exiqon (Denmark). Hybridization and washing were performed as described by Válóczi [36]. Signals were detected using CDP-Star chemiluminescent substrate (Roche), and the blot was exposed to the Lumi-Imager F1 Workstation. To control equal loading, blots were hybridized with a DIG-labeled probe against U6 snRNA.

\section{In situ hybridization}

Ascidian embryos, larvae, and juveniles at 21, 34, and 42 hpf were collected and fixed in $4 \%$ paraformaldehyde (PFA) overnight at $4{ }^{\circ} \mathrm{C}$. DIG-labeled LNA-modified probes (Additional file 5: Table S3) were designed and synthesized by Exiqon (Denmark). Whole-mount in situ hybridization was performed as previously described [37] at a temperature $20{ }^{\circ} \mathrm{C}$ below the probe melting temperature. Briefly, after fixation, samples were placed in PBST and washed four times at room temperature. Then, samples were treated with proteinase $\mathrm{K}$ at $37{ }^{\circ} \mathrm{C}$ for $20 \mathrm{~min}$. After treatment, samples were re-fixed in $4 \%$ PFA and washed four times in PBST. After washing, samples were pre-hybridized in a prehybridization buffer for $2 \mathrm{~h}$ in a humid chamber. Subsequently, hybridization was conducted at hybridization temperature for $18 \mathrm{~h}$ using miRNA LNA probes in a hybridization solution. After hybridization, samples were washed in gradient saline-sodium citrate at the hybridization temperature. Signals of hybridization were detected using alkaline phosphatase-conjugated digoxigenin antibody (Roche) at a 1:2000 dilution. Samples were stained with BCIP/NBT and visualized under a microscope.

\section{Bioinformatics analysis}

Clean data (clean reads) were obtained by removing reads containing poly- $\mathrm{N}$, with 5 'adapter contaminants, without 3 ' adapter or the insert tag, containing poly A or $\mathrm{T}$ or $\mathrm{G}$ or $\mathrm{C}$ and low-quality reads from raw data. miRDeep2 [38] and miReap (http://sourceforge.net/projects/ mireap/) were then utilized to identify miRNAs by mapping clean sRNA reads to predict pre-miRNA structures in the C. savignyi genome. Novel and conserved miRNAs were then classified by searching for them against miRBase20.0 (http://mirbase.org/). For miRNA-target prediction, 3'-UTRs of C. savignyi mRNAs obtained from the ENSEMBL database were used as potential target sequences. miRNA binding sites were then predicted using miRanda [39] and TargetScan 7.1 (targetscan.org). Targets predicted using miRanda whose expression levels on performing RNA-Seq were negatively correlated with miRNAs were collected for further analysis. The interactions of miRNA and their target genes were visualized as a network using Cytoscape3.4. KOBAS software [40] was utilized to test the statistical enrichment of target gene candidates in KEGG pathways.

For evolutionary analysis, C. savignyi miRNAs were compared with miRNAs of 16 other animal species downloaded from miRBase. Only miRNAs supported by experimental evidence were used. The mature miRNAs sequences from different species were clustered according to sequence similarity, to identify conserved miRNA families, requiring alignment $\geqq 15$ nt which covers the miRNA seed region (position 2-8 nt) and mismatch $\leqq 2$. Moreover, at most $1 \mathrm{nt}$ overhang was allowed in the alignment between the 5 'ends of mature miRNAs. Then reconstruction of the evolution history of miRNA families were performed using in-house perl scripts, applying the Dollo parsimony criteria.

\section{Cell culture}

Human embryonic kidney 293T cells (HEK293T) were cultured in DMEM (Invitrogen, USA) supplemented with $10 \%$ fetal bovine serum (Gibco, USA), penicillin $(100 \mathrm{U} / \mathrm{ml})$, and streptomycin $(100 \mu \mathrm{g} / \mathrm{ml})$ in a humidified atmosphere of $5 \% \mathrm{CO}_{2}$ at $37{ }^{\circ} \mathrm{C}$. 


\section{Luciferase reporter constructs and luciferase assay} Wild-type C. savignyi MAPK1-3'-UTR or MAPKK33 '-UTR fragments containing predicted miRNA target sites were amplified by PCR using C. savignyi embryo cDNA as templates. A mutant 3'-UTR construct (pmirGLO-MAPK1-3' -UTR mutant) with a mutation of three nucleotides (Fig. 7a) from the site of MAPK1 or pmirGLO-MAPKK3-3'-UTR mutant with a mutation of seven nucleotides (Additional file 4: Figure S2A) from the site of the MAPKK3 gene were generated by overlap extension PCR. Primer sequences are shown in Additional file 6: Table S4. Both wild-type and mutant 3 '-UTRs were cloned into the firefly luciferase coding region of the pmirGLO plasmid (Promega, USA).

For reporter assays, cell transfections were performed using Lipofectamine 3000 (Invitrogen, USA) following the manufacturer's instructions. One day before transfection, HEK293 cells were passaged in 24-well plates at 70-80\% confluence. Then, $0.4 \mu \mathrm{g}$ of miRNA 3'-UTR plasmid, $1 \mu \mathrm{l}$ of $\mathrm{P} 3000$ regent, and $100 \mathrm{nM}$ miRNA mimics (Genepharma, China) were diluted in $25 \mu \mathrm{l}$ of medium and $1.5 \mu \mathrm{l}$ of Lipofectamine 3000 was diluted in $25 \mu \mathrm{l}$ of medium. Then, the diluted construct was mixed with the diluted Lipofectamine 3000. The mixture was incubated for $5 \mathrm{~min}$ at room temperature and was then added to each well containing $500 \mu \mathrm{l}$ of cells and the medium. Forty-eight hours after transfection, firefly and Renilla luciferase activities were measured using the Dual-Luciferase reporter assay system (Promega, USA), and relative reporter activity was normalized to Renilla luciferase activity. Each assay was performed in triplicate.

\section{Conclusions}

We identified 165 miRNAs, including 59 novel ones, from the embryos and larvae of $C$. savignyi. Twelve of them showed significant changes in expression before and during metamorphosis. In situ hybridization and northern blotting results revealed that three miRNAs are potentially involved in the signaling regulatory network for the migration and differentiation of mesenchymal cells in larval metamorphosis. We also revealed the target genes of identified 12 miRNAs. Our results not only present a list and profile of miRNAs involved in Ciona metamorphosis but also provide informative cues to further understand their function in ascidian larval metamorphosis.

\section{Additional files}

Additional file 1: Table S1. Sequences of identified known miRNAs in C. savignyi. (DOCX $108 \mathrm{~kb}$ )

Additional file 2: Table S2. Sequences of identified novel miRNAs in C. savignyi. (DOCX $103 \mathrm{~kb}$ )
Additional file 3: Figure S1. Original images of northern blotting presented in Fig. 5. (PDF $1785 \mathrm{~kb}$ )

Additional file 4: Figure S2. Validation of the interaction between csa-miR-4018a or csa-miR-4018b and Mapkk3-3'-UTR. (PDF 841 kb)

Additional file 5: Table S3. Sequences of probes used in northern blotting and in situ hybridization. (DOCX 47 kb)

Additional file 6: Table S4. Sequence of primers for mutant constructs used in overlap extension PCR. (DOCX $60 \mathrm{~kb}$ )

\section{Abbreviations}

EGF: Epithelial growth factor; ERK: Extracellular regulated protein kinase; JNK: C-Jun N-terminal kinase; LNA: Locked nucleic acid; MAPK: Mitogenactivated protein kinase; MKK: Mitogen-activated protein kinase kinase; MKP: Mitogen-activated protein kinase phosphatase; MSCs: Mesenchymal stem cells; PFA: Paraformaldehyde; Rap: Ras-related protein; TGF- $\beta$ : Transforming growth factor beta

\section{Acknowledgments}

Not applicable.

\section{Funding}

This work was supported by the National Natural Science Foundation of China (Grant No. 31572352, 41706153, 31771649), Qingdao National Laboratory for Marine Science and Technology (QNLM2016ORP0301), and the Taishan Scholar Program of Shandong Province, China (201502035).

\section{Availability of data and materials}

The datasets supporting the conclusions of this article are included within the article and its additional files.

\section{Authors' contributions}

XMZ performed the experiments, analyzed the data, drafted the figures, and prepared the manuscript; XZL performed the experiments; CZL analyzed the data and prepared part of the manuscript; JKW analyzed RNA-Seq data for target gene prediction; HYY provided the reagents for experiments and advice on data analysis; BD designed and monitored the experiments, and drafted and revised the manuscript. All authors have read and approved the final manuscript.

\section{Ethics approval and consent to participate}

All of the procedures involved in the handling and treatment of C. savignyi in this study were approved by the Ocean University of China Institutional Animal Care and Use Committee (OUC-IACUC) prior to the initiation of the study. And all experiments and relevant methods were carried out in accordance with the approved guidelines and regulations of OUC-IACUC.

\section{Consent for publication}

Not applicable.

Competing interests

The authors declare that they have no competing interests.

\section{Publisher's Note}

Springer Nature remains neutral with regard to jurisdictional claims in published maps and institutional affiliations.

\section{Author details}

${ }^{1}$ Ministry of Education Key Laboratory of Marine Genetics and Breeding, College of Marine Life Sciences, Ocean University of China, Qingdao 266003, China. ${ }^{2}$ Laboratory for Marine Biology and Biotechnology, Qingdao National Laboratory for Marine Science and Technology, Qingdao 266237, China. ${ }^{3}$ Key Laboratory of Experimental Marine Biology, Institute of Oceanology, Chinese Academy of Sciences, Qingdao 266071, China. ${ }^{4}$ Institute of Evolution and Marine Biodiversity, Ocean University of China, No. 5 Yushan Road, Qingdao 266003, People's Republic of China. 
Received: 9 September 2017 Accepted: 22 February 2018 Published online: 01 March 2018

\section{References}

1. Hirano T, Nishida H. Developmental fates of larval tissues after metamorphosis in ascidian Halocynthia roretzi. I. Origin of mesodermal tissues of the juvenile. Dev Biol. 1997;192(2):199-210.

2. Woods RG, Roper KE, Gauthier M, Bebell LM, Sung K, Degnan BM, Lavin MF. Gene expression during early ascidian metamorphosis requires signalling by hemps, an EGF-like protein. Development. 2004;131(12):2921-33.

3. Davidson B, Swalla BJ. Isolation of genes involved in ascidian metamorphosis: epidermal growth factor signaling and metamorphic competence. Dev Genes Evol. 2001;211(4):190-4.

4. Nakayama A, Satou Y, Satoh N. Isolation and characterization of genes that are expressed during Ciona intestinalis metamorphosis. Dev Genes Evol. 2001;211(4):184-9.

5. Chambon J-P, Nakayama A, Takamura K, McDougall A, Satoh N. ERK- and JNK-signalling regulate gene networks that stimulate metamorphosis and apoptosis in tail tissues of ascidian tadpoles. Development. 2007;134(6): 1203-19.

6. Tarallo R, Sordino P. Time course of programmed cell death in Ciona intestinalis in relation to mitotic activity and MAPK signaling. Dev Dyn. 2004 230(2):251-62

7. Comes S, Locascio A, Silvestre F, Ischia M, Russo GL, Tosti E, Branno M, Palumbo A. Regulatory roles of nitric oxide during larval development and metamorphosis in Ciona intestinalis. Dev Biol. 2007:306(2):772-84.

8. Castellano I, Ercolesi E, Palumbo A. Nitric oxide affects ERK signaling through down-regulation of MAP kinase phosphatase levels during larval development of the ascidian Ciona intestinalis. PLoS One. 2014;9(7):e102907.

9. Aranha MM, Santos DM, Xavier JM, Low WC, Steer CJ, Solá S, Rodrigues CMP. Apoptosis-associated microRNAs are modulated in mouse, rat and human neural differentiation. BMC Genomics. 2010;11(1):514.

10. Thompson BJ, Cohen SM. The hippo pathway regulates the bantam microRNA to control cell proliferation and apoptosis in drosophila. Cell. 2006:126(4):767-74.

11. Cimmino A, Calin GA, Fabbri M, lorio MV, Ferracin M, Shimizu M, Wojcik SE, Ageilan Rl, Zupo S, Dono M, et al. miR-15 and miR-16 induce apoptosis by targeting BCL2. Proc Natl Acad Sci. 2005;102(39):13944-9.

12. Gomez-Orte E, Belles X. MicroRNA-dependent metamorphosis in hemimetabolan insects. Proc Natl Acad Sci. 2009;106(51):21678-82.

13. Caygill $E E$, Johnston LA. Temporal regulation of metamorphic processes in drosophila by the let-7 and miR-125 heterochronic microRNAs. Curr Biol. 2008;18(13):943-50.

14. Fu X, Adamski M, Thompson EM. Altered miRNA repertoire in the simplified chordate, Oikopleura dioica. Mol Biol Evol. 2008;25(6):1067-80.

15. Shi W, Hendrix D, Levine M, Haley B. A distinct class of small RNAs arises from pre-miRNA-proximal regions in a simple chordate. Nat Struct Mol Biol. 2009:16(2):183-9.

16. Hendrix D, Levine $M$, Shi W. miRTRAP, a computational method for the systematic identification of miRNAs from high throughput sequencing data. Genome Biol. 2010;11(4):R39.

17. Velandia-Huerto CA, Gittenberger AA, Brown FD, Stadler PF, BermúdezSantana $\mathrm{Cl}$. Automated detection of ncRNAs in the draft genome sequence of a colonial tunicate: the carpet sea squirt Didemnum vexillum. BMC Genomics. 2016;17(1):691.

18. Wang K, Dantec C, Lemaire P, Onuma TA, Nishida H. Genome-wide survey of miRNAs and their evolutionary history in the ascidian, Halocynthia roretzi. BMC Genomics. 2017;18(1):314.

19. Keshavan R, Virata M, Keshavan A, Zeller RW. Computational identification of Ciona intestinalis microRNAs. Zool Sci. 2010;27(2):162-70.

20. Chen JS, Pedro MS, Zeller RW. miR-124 function during Ciona intestinalis neuronal development includes extensive interaction with the notch signaling pathway. Development. 2011;138(22):4943-53.

21. Di Felice $V$, Zummo G. Stem cell populations in the heart and the role of Isl1 positive cells. Eur J Histochem. 2013;57(2):e14.

22. Lim LS, Hong FH, Kunarso G, Stanton LW. The pluripotency regulator Zic3 is a direct activator of the Nanog promoter in ESCs. Stem Cells. 2010;28(11):1961-9.

23. Minamino Y, Ohnishi Y, Kakudo K, Nozaki M. Isolation and propagation of neural crest stem cells from mouse embryonic stem cells via cranial neurospheres. Stem Cells Dev. 2015;24(2):172-81.
24. Wheeler BM, Heimberg AM, Moy VN, Sperling EA, Holstein TW, Heber S, Peterson KJ. The deep evolution of metazoan microRNAs. Evol Dev. 2009; 11(1):50-68

25. Peterson KJ, Dietrich MR, McPeek MA. MicroRNAs and metazoan macroevolution: insights into canalization, complexity, and the Cambrian explosion. BioEssays. 2009;31(7):736-47.

26. Nakayama-Ishimura A, Chambon J-P, Horie T, Satoh N, Sasakura Y. Delineating metamorphic pathways in the ascidian Ciona intestinalis. Dev Biol. 2009;326:357-67.

27. Alexander M, Hu R, Runtsch MC, Kagele DA, Mosbruger TL, Tolmachova T, Seabra MC, Round JL, Ward DM, O'Connell RM. Exosome-delivered microRNAs modulate the inflammatory response to endotoxin. Nat Commun. 2015:6:7321.

28. Umezu T, Ohyashiki K, Kuroda M, Ohyashiki JH. Leukemia cell to endothelial cell communication via exosomal miRNAs. Oncogene. 2013:32(22):2747-55.

29. Lee HK, Finniss S, Cazacu S, Xiang C, Brodie C. Mesenchymal stem cells deliver exogenous miRNAs to neural cells and induce their differentiation and glutamate transporter expression. Stem Cells Dev. 2014;23(23):2851-61.

30. Kim GJ, Nishida H. Role of the FGF and MEK signaling pathway in the ascidian embryo. Dev Growth Differ. 2001:43(5):521-33.

31. Kim GJ, Kumano G, Nishida H. Cell fate polarization in ascidian mesenchyme/muscle precursors by directed FGF signaling and role for an additional ectodermal FGF antagonizing signal in notochord/nerve cord precursors. Development. 2007;134(8):1509-18.

32. Nishida H. Spatio-temporal pattern of MAP kinase activation in embryos of the ascidian Halocynthia roretzi. Develop Growth Differ. 2003:45(1):27-37.

33. Hayashi T, Lombaert IMA, Hauser BR, Patel VN, Hoffman MP. Exosomal MicroRNA transport from salivary mesenchyme regulates epithelial progenitor expansion during organogenesis. Dev Cell. 2017;40(1):95-103.

34. Phinney DG, Di Giuseppe M, Njah J, Sala E, Shiva S, St Croix CM, Stolz DB, Watkins SC, Di YP, Leikauf GD, et al. Mesenchymal stem cells use extracellular vesicles to outsource mitophagy and shuttle microRNAs. Nat Commun. 2015:6:8472

35. Dambal S, Shah M, Mihelich B, Nonn L. The microRNA-183 cluster: the family that plays together stays together. Nucleic Acids Res. 2015;43(15): 7173-88.

36. Válóczi A, Hornyik C, Varga N, Burgyán J, Kauppinen S, Havelda Z Sensitive and specific detection of microRNAs by northern blot analysis using LNAmodified oligonucleotide probes. Nucleic Acids Res. 2004;32(22):e175.

37. Christiaen L, Wagner E, Shi W, Levine M. Whole-mount in situ hybridization on sea squirt (Ciona intestinalis) embryos. Cold Spring Harb Protoc. 2009; 2009(12):pdb.prot5348.

38. Friedländer MR, Mackowiak SD, Li N, Chen W, Rajewsky N. miRDeep2 accurately identifies known and hundreds of novel microRNA genes in seven animal clades. Nucleic Acids Res. 2012;40(1):37-52.

39. Enright AJ, John B, Gaul U, Tuschl T, Sander C, Marks DS. MicroRNA targets in drosophila. Genome Biol. 2004;5(1):14

40. Mao X, Cai T, Olyarchuk JG, Wei L. Automated genome annotation and pathway identification using the KEGG Orthology (KO) as a controlled vocabulary. Bioinformatics. 2005;21(19):3787-93.

\section{Submit your next manuscript to BioMed Central and we will help you at every step:}

- We accept pre-submission inquiries

- Our selector tool helps you to find the most relevant journal

- We provide round the clock customer support

- Convenient online submission

- Thorough peer review

- Inclusion in PubMed and all major indexing services

- Maximum visibility for your research

Submit your manuscript at www.biomedcentral.com/submit 